\title{
Effect of Yoga on Primary Dysmenorrhea and Stress in Medical Students
}

\author{
Usha Nag, M.D, [Obg], Pg Dip, M.Sc [Yoga] ${ }^{1}$ Madhavi Kodali, M.D.,D.P.M. ${ }^{2}$ \\ ${ }^{1}$ Associate Professor, Department of OBG, Dr. Pinnamaneni Siddhartha Institute Of Medical Sciences And \\ Research Foundation, India. \\ ${ }^{2}$ Assistant Professor, Department of Psychiatry, Dr. Pinnamaneni Siddhartha Institute Of Medical Sciences And \\ Research Foundation, India.
}

\begin{abstract}
Objective: Few studies have been done on yoga as therapy for stress and primary dysmenorrhoea. This study is aimed at evaluating the efficacy of yoga as alternative therapy for primary dysmenorrhoea and stress.

Methods:113 medical students, unmarried girls, with primary dysmenorrhoea and stress, were randomly assigned to study $(n=60)$ and control group $(n=53)$. Semi structured questionnaire, the Numerical rating scale for pain and the Perceived Stress Scale were administered at baseline and after three months. The study group was subjected to yoga intervention.

Results: Significant $(p<0.0001)$ reduction in the perceived pain after yoga intervention in study group. $88 \%$ of the study group reported complete pain relief and $12 \%$ reported mild pain. Compared to control group, students with yoga intervention demonstrated significant improvement in perceived stress $(P<0.0001) .82 \%$ of the study group reported complete stress relief. After yoga intervention, absenteeism dropped to $10 \%$ and improvement in daily activity was observed in study group.

Conclusion: With yogic exercises the stress and pain of dysmenorrhoea was controlled indicating the benefits of yoga in primary dysmenorrhoea. Yoga lessens psychosocial stress levels, so it should be implemented among college students to augment their menstrual well-being.
\end{abstract}

Key words: dysmenorrhoea, stress, yoga

\section{Introduction}

Menstrual disorders are a common presentation by late adolescence, $75 \%$ of girls experience some problems associated with menstruation ${ }^{1}$. Dysmenorrhoea is a common problem in women of reproductive age. Primary dysmenorrhoea is defined as painful menses in women with normal pelvic anatomy, usually begins during adolescence ${ }^{2}$. Affected women experience sharp, intermittent spasm of pain usually concentrated in the supra pubic area. Pain may radiate to the back of the legs or the lower back. Systemic symptoms of nausea, vomiting, diarrhoea, fatigue, mild fever and headache or light headedness are fairly common. Pain usually develops within hours of the start of the menstruation and peaks as the flow becomes heaviest during the first day or two of the cycle. ${ }^{3}$

Primary Dysmenorrhoea is the most common gynaecologic disorder among female adolescents, with a prevalence of $60 \%$ to $93 \%$. Several studies have shown that adolescents with primary dysmenorrhoea report that it effects their academic performance, social and sports activities and is a cause for school absenteeism. ${ }^{1,4}$ The most common effect of menstrual problems on daily routine, reported by unmarried undergraduate medical students was in the form of prolonged resting hours followed by inability to study. ${ }^{5}$ The aetiology of primary dysmenorrhoea is not precisely understood, but most symptoms can be explained by the action of uterine prostaglandins, particularly PGF2- Alfa. ${ }^{6}$ The risk factors for dysmenorrhoea are; age $<20$ years, nulliparity, heavy menstrual flow, smoking, upper socioeconomic status; attempts to lose weight, physical inactivity, disruption of social networks, depression and anxiety ${ }^{7}$. Physical activity is also an important behavioral cofactor; people who describe themselves as active have lower levels of inflammatory biomarkers than their sedentary counterparts ${ }^{8}$.

Additionally, psychological stressors can directly provoke transient increases in proinflammatory cytokines. ${ }^{9}$ Yoga's reputation for stress reduction and mental health benefits has bolstered its popularity in recent years, and data from randomized trials suggest that yoga reduces symptoms of anxiety and depression ${ }^{10}$. Mechanistic explanations for yoga's potential mental and physical health benefits have highlighted reductions in sympathetic nervous system tone and increases in vagal activity, both of which could have favourable endocrine and immune consequences, including lower inflammation ${ }^{11,12}$. It has been suggested that the number of regular ovulatory menstrual cycles is associated with an increased risk of breast cancer for women ${ }^{13}$. At the same time, there is growing evidence of an association between psychosocial stress and menses-associated health problems in women, suggesting that stress may affect menstrual function. ${ }^{14}$ 
However, Studies to date have examined the relation between stress and the risk of dysmenorrhoea. ${ }^{14}$ Yoga appears to provide improvement in stress. ${ }^{15,16,17,18}$ Very few studies suggested that yoga reduced the severity and duration of primary dysmenorrhea. ${ }^{19}$

In this above back ground the present study has been undertaken with the aim to evaluate the efficacy of yoga in relieving the menstrual pain and stress in unmarried girls.

\section{Methods}

This study was carried out at Dr. Pinnamaneni Siddhartha Institute of Medical Sciences from July to October 2010. All the girl students of our medical college $\left[1-5^{\text {th }}\right.$ year $]$ were screened for primary dysmenorrhoea and stress using a structured questionnaire and 4-item version of The Perceived stress scale. Unmarried girl students, within the age group 18-23 years, with primary dysmenorrhoea and stress were included in our study. Students with diagnosed psychiatric disorders would have been excluded from the study but no participant met this criteria. Students already practicing yoga and with other causes of secondary dysmenorrhea were excluded from the study. Out of the 320 girl medical students 113 students fulfilled the fixed criteria. These subjects were assured of confidentiality of information after explaining the purpose of the study and obtaining consent. Of the 113 student who fulfilled the fixed criteria, sixty subjects were selected by simple random sampling and were allotted to the study group $(n=60)$ for yoga intervention and the remaining $(n=53)$ were included in the control group. Semi structured proforma, the Numerical rating scale (NRS) for assessment of intensity of pain and The Perceived stress scale (PSS) for stress assessment were administered to the students of both study and control group at the baseline. The 60 participants in the study group were asked to attend 60 minute yoga class every day for a period of 3 months taught by the Yoga instructor. All classes were free of charge to the participants. The control group $(n=53)$ did not receive any intervention and were asked to complete the questionnaires. Each group was evaluated after three months. Semi structured questionnaire, Numerical rating scale (NRS) and The Perceived stress scale (PSS) were administered on both the groups at the end of three months.

Yoga intervention: The following Yoga poses were done by the study group: Navasana, Matsyasana, Dhanurasana, Vajrasana, Paschimotasana, Ustrasana, Ardhamatstyendrasana, Salabhasana, Bhujangasana, Sarvangasana, Uttanapadasana, Padmasana, and Surya namaskara (24 times).

Semi structured proforma consists the details of the socioeconomic status; detailed history of menstrual flow; menstrual cycle; premenstrual symptoms; menstrual pain; college absenteeism and involvement in daily activities during menstruation.

Numerical rating scale (NRS) ${ }^{30}$ typically consists of a range of numbers from (0-10) from which the patient selects the number most representative of their pain. 0 would mean 'No pain' and 10 would mean 'Worst possible pain'.

The Perceived Stress scale (PSS) is a validated self-report questionnaire widely used for assessing an individual's self-perception of stress. The PSS has 14-, 10- and 4-item versions and has been shown to yield adequate reliability and validity (Cohen et al., 1983; Cohen and Williamson, 1988). In this study, 4-item version was used to screen potential subjects while the 10 -item version was used to assess change in perceived stress before and after 3 months. Participants gave their responses on a 5-point Likert scale, ranging from never (0) to very often (4).

Statistical analysis was done with SPSS 11.5 and the results were expressed in proportions. Friedman test were applied to find out the significance of association and $\mathrm{p}$ value $<0.05$ was considered as statistically significant.

\section{Results}

In the present study 113 medical students, unmarried girls, with primary dysmenorrhoea and stress, were recruited and randomly assigned to study group $(n=60)$ and a control group $(n=53)$. The baseline characteristics were very similar for the study and the control groups. All the participants in this study completed the questionnaires at the beginning of the study and at the end of three months. Semi structured questionnaire, The Numerical rating scale for pain and The Perceived Stress Scale were administered on all the participants. The subjects of the study group were asked to do yoga poses and the control group did not receive any intervention. We found that most of the participants (75\%) suffering from both dysmenorrhoea and stress belong to high socioeconomic status.

In the present study we observed that in $98 \%$ of the study group and $57 \%$ of the control group the involvement in daily activities was affected and college absenteeism was more. After 3 months of yoga intervention absenteeism was reported only in $10 \%$ of the study group. Improvement in the daily activity involvement was also observed and only $10 \%$ of study group reported with disturbance.

Figure1 shows the comparison of PSS scores before and after yoga intervention in the study group. Of the 60 (study group) 9 subjects were in slightly higher than average category. 51 subjects were in the much 
higher than average category. Thus, $85 \%$ of the study group perceived much higher than average stress. After three months of yoga intervention 49 subjects $(82 \%)$ reported complete stress relief, 8 subjects reported average stress and 3 reported slightly higher than average stress. We observed significant $(p<0.0001)$ improvement in the stress levels after three months of yoga intervention.

Figure 2 shows the PSS scores at the baseline and after three months in the control group. 7 subjects of the control group $(n=53)$ reported slightly higher than average stress and 46 subjects reported much higher than average stress at the baseline. At the end of three months 50 subjects (94\%) did not show any change in the stress level.

Figure 3 shows the comparison of NRS scores before and after yoga intervention in the study group. Out of the 60 study group 2 reported with mild pain, 47 reported with moderate pain and 11 reported with severe pain. At the end of three months of yoga intervention 53 subjects (88\%) reported with complete pain relief and 7 subjects reported with mild pain. We observed that there was significant $(\mathrm{p}<0.0001)$ reduction in the pain perceived after yoga intervention.

Figure 4 shows the NRS scores at the baseline and after three months in the control group. 44 subjects of the control group $(n=53)$ reported mild to moderate pain and 9 subjects reported severe pain. At the end of three months no change was observed in 52 subjects.

At the end of three months of yoga intervention the PSS scores of the study group and control group were compared and analysed using Friedman test and the $\mathrm{p}$ value was found to be $<0.0001$ which is highly significant statistically. NRS scores were compared between the study and control group at the end of the study and p value $(<0.0001)$ was found statistically significant.

\section{Discussion}

In the present study, we examined the efficacy of yoga in relieving the menstrual pain and stress in unmarried female college students. In the present study we observed that in $98 \%$ of the study group and $57 \%$ of the control group the involvement in daily activities was affected and college absenteeism was more. Similar results have been reported by Sharma et al. ${ }^{5}$ However, after 3 months of yoga intervention, absenteeism came down to $10 \%$ in the study group. There was also improvement in the daily activity.

Students who had menstrual pain had higher stress scores than those who did not have menstrual pain, stress score was found to be associated with menstrual pain in the current study. Wang $L$ et al suggested that perceived stress was associated with painful menses ${ }^{15}$. Regardless of these facts, it has been shown that daily stressors or perceived stress appears to be associated with premenstrual symptoms and that stress reducing measures such as eliciting a relaxation response and aerobic exercise are effective in alleviating premenstrual symptoms ${ }^{21}$. The current results seem to be consistent with these earlier findings. In our study compared to control group, students who had yoga intervention for three months demonstrated pronounced and significant improvements in perceived stress $(\mathrm{P}<0.0001) .82 \%$ (49 subjects) of the study group reported complete stress relief.

We observed after yoga intervention in study group there is significant $(p<0.0001)$ reduction in the perceived pain using Friedman test. 88\% of the study group (53 subjects) reported with complete pain relief and $12 \%$ (7 subjects) reported with mild pain. In contrast, no reduction of pain was found in the control group. NRS scores were compared between the study and control group at the end of the study and p value $(<0.0001)$ was found statistically significant.

Our results are comparable with Rakhshaee Z, who suggested that yoga reduced the severity and duration of primary dysmenorrhea. ${ }^{19}$ In her study it was found that there were significant differences in pain intensity with using Friedman test and in pain duration using with repeated measure ANOVA test in the two post-tests compared with the pretest $(P=0.000) .{ }^{19}$ This means that intensity and duration of dysmenorrhea had been reduced after doing yoga. Also, after doing yoga in second month, pain intensity and pain duration reduced more with comparison to the first month. ${ }^{19} \mathrm{~A}$ significant difference was observed between experimental and control groups in pain intensity and pain duration with using Mann-Whitney test. ${ }^{19}$

Differences in stress responses between yoga performers and non performers provided one plausible mechanism for their divergent serum IL-6 data; Yoga performers produced less LPS-stimulated IL-6 in response to the stressor than the non performers ${ }^{22}$. Some hatha yoga postures are characterized as immune enhancing or restorative $^{23}$. Psychosocial stress may be a factor responsible for the derangement of menstrual cycles, thereby predisposing women under psychosocial stress to long-term disease risks. Taken together with these findings, our results lead us to the conclusion that more attention should be paid to regimens which may alleviate psychosocial stress in order to augment the well-being of female students and which will potentially contribute to reducing their future risks for diseases such a type 2 diabetes, cardiovascular disease, and infertility.

The current study has several limitations. First, this study was based on a self-administered questionnaire. However, the bias seems to be minimal as the proportion of students who had menstrual pain in 
our study was similar to those in earlier reports. Second, this study was conducted in the rather restricted study population of medical students, thus generalization of the current findings may be limited.

\section{Conclusion:}

The present results suggest that psychosocial stress is independently associated with menstrual pain among unmarried women of reproductive age and Yoga has been found to be an effective alternative method to produce considerable pain relief in primary dysmenorrhoea. With yogic exercises the stress and pain of dysmenorrhoea was controlled indicating the benefits of yoga in primary dysmenorrhoea. Alternative Measures like yoga that may lessen psychosocial stress levels should be implemented among college students in order to augment their menstrual well-being, thereby potentially preventing future illness from occurring.

The Principal, Dr. PSIMS \& RF.

\section{Acknowledgement:}

Mr. Chari MSc. (Statistics), Mrs. Komala Rani.

All subjects of the study for their co operation in the successful completion of the study.

\section{References}

[1] Lee LK, Chen PCY, Lee KK, Kaur J. Menstruation among adolescent girls in Malaysia: a cross-sectional school survey. Singapore Med J 2006; 47(10): 874.

[2] Campbell M, McGrath P. Use of medication by adolescents for the management of menstrual discomfort. Arch Pediatr Adolesc Med 1997; 151: 905-912.

[3] Alvin P, Litt I . Current status of the etiology and the management of dysmenorrhea in adolescence. Pediatrics 1982; 70: 516-525.

[4] Banikarim C, Chacko MR, Kelder SH. Prevalence and impact of dysmenorrhea on Hispanic female adolescents. Arch Pediatr Adolesc Med 2000; 154: 1226-1229.

[5] Sharma A, Taneja DK, Sharma P. Problems related to menstruation and their effect on daily routine of students of a medical college in Delhi, India. Asia Pac J Public Health. 2008;20(3):234-41.

[6] Andersch B, Milsom I. An epidemiologic study of young women with dysmenorrhea. Am J Obstet Gynecol 1982; 144: 655-660.

[7] Cakir M, Mungan I, Karakas T, Girisken I, Okten A. Menstrual pattern and common menstrual disorders among university students in Turkey. Pediatr Int 2007 Dec; 49(6): 938-942.

[8] Hamer M. The relative influences of fitness and fatness on inflammatory factors. Prev Med 2007;44:3-11.

[9] Steptoe A,Hamer M, Chida Y. .The effects of acute psychological stress on circulating inflammatory factors in humans: A review and meta-analysis. Brain Behavior and Immunity 2007;21:901-912.

[10] Pilkington K, Kirkwood G, Rampes H, Richardson J Yoga for depression: The research evidence. J Affect Disord 2005;89:13-24.

[11] Riley D. Hatha yoga and the treatment of illness. Altern Ther Health Med 2004; 10:20-21.

[12] Bernardi L, Sleight P, Bandinelli G, Cencetti S, Fattorini L, Wdowczyc-Szulc J, Lagi A.. Effect of rosary prayer and yoga mantras on autonomic cardiovascular rhythms: comparative study. Br Med J 2001; 323:1446-1449.

[13] Henderson BE Ross RK, Judd HL, Krailo MD, Pike MC. Do regular ovulatory cycles increase breast cancer risk? Cancer 1985;56: 1206-1208

[14] Gordley LB, Lemasters G, Simpson SR, Yiin JH. Menstrual disorders and occupational stress, and racial factors among military personnel. J Occup Environ Med 2000; 42: 871-881

[15] Wang L, Wang X, Wang W, Chen C, Ronnennberg AG, Guang W, Huang A, Fang Z, Zang T, Wang L, Xu X Stress and dysmenorrhoea: a population based prospective study. Occup Environ Med 2004; 61: 1021-1026

[16] Smith C, Hancock H, Blake-Mortimer J, Eckert K. A randomised comparative trial of yoga and relaxation to reduce stress and anxiety. Complement Ther Med. 2007 Jun;15(2):77-83.

[17] White LS. Reducing stress in school-age girls through mindful yoga. J Pediatr Health Care. 2012 Jan-Feb;26(1):45-56.

[18] Parshad O. Role of yoga in stress management. West Indian Med J. 2004 Jun;53(3):191-4.

[19] Rakhshaee Z. Effect of three yoga poses (cobra, cat and fish poses) in women with primary dysmenorrhea: a randomized clinical trial. J Pediatr Adolesc Gynecol. 2011 Aug;24(4):192-6.

[20] McCaffery, M.,Beebe, A. (1993). Pain: Clinical Manual for Nursing Practice. Baltimore: V.V. Mosby Company

[21] Steege JF, Blumenthal JA. The effects of aerobic exercise on premenstrual symptoms in middle-aged women:a preliminary study. J Psychosom Res 1993; 37: 127-133

[22] Janice K. Janice K. Kiecolt-Glaser, Phd, Lisa Christian, Phd, Heather Preston, Ba, Carrie R. Houts, Ms Stress, Inflammation, and Yoga Practice, Psychosomatic Medicine 2010; 72:113-121

[23] Iyengar, BKS. Light on yoga. New York: Schocken Books; 1995. 
Figures:

Figure1: Comparison of PSS scores before and after yoga intervention in the study group.

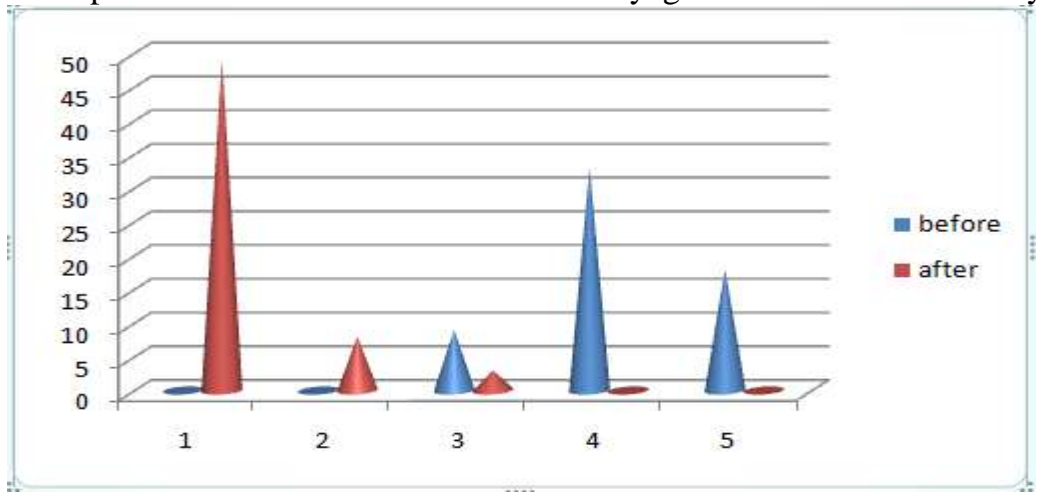

Figure 2: PSS scores at the baseline and after three months in the control group.

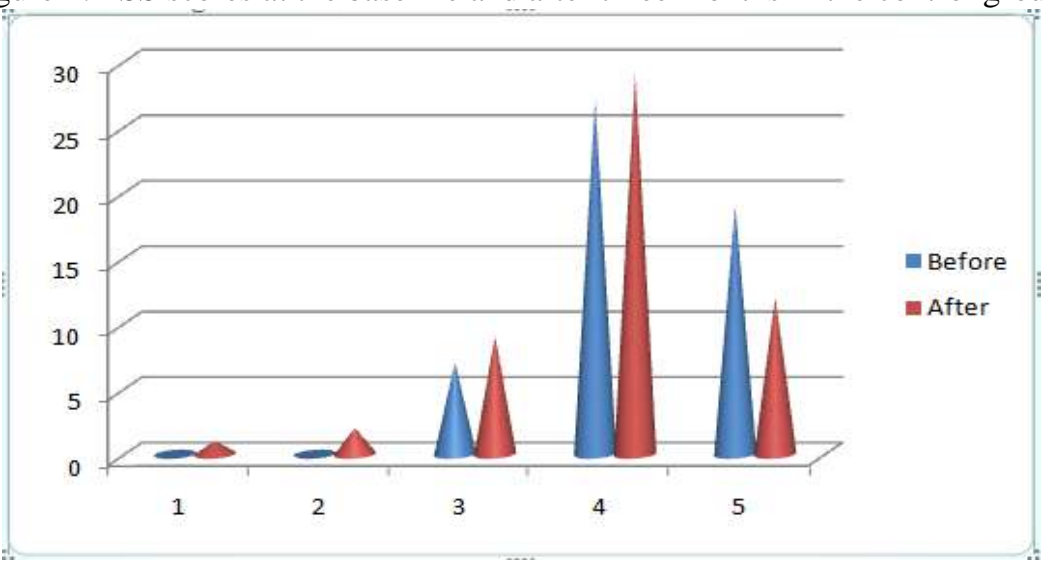

Figure 3: Comparison of NRS scores before and after yoga intervention in the study group

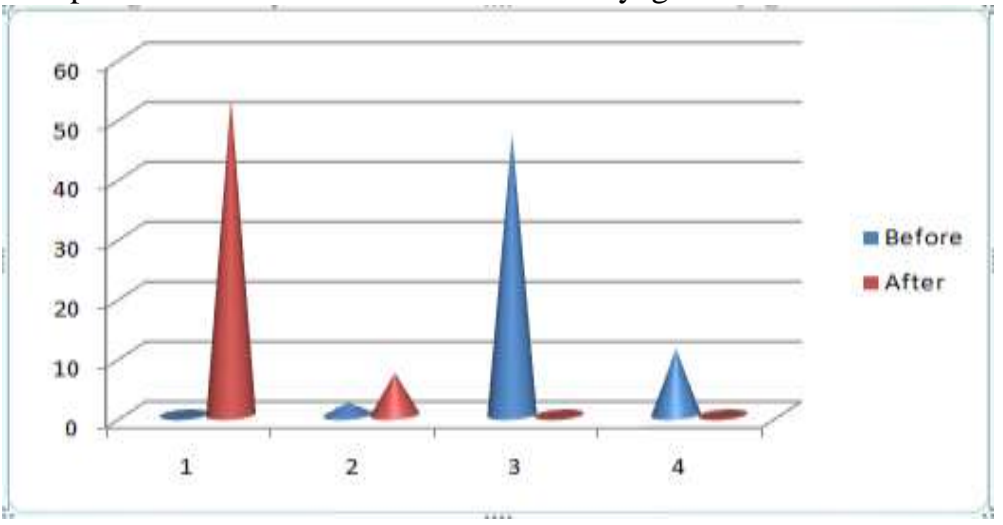

Figure 4: NRS scores at the baseline and after three months in the control group.

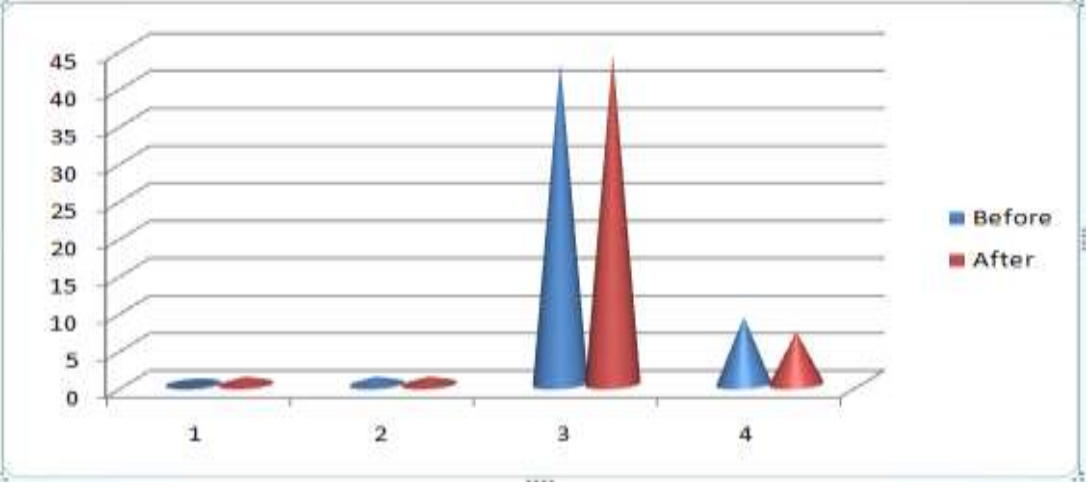

